\title{
A case report of eosinophilic gastroenteritis misdiagnosed as cholelithiasis
}

\author{
qianlong $\mathrm{li}^{1}$, suyu he ${ }^{1}$, tianyu liu ${ }^{1}$, lianfen $\mathrm{h}^{1}$, yingdong $\mathrm{jia}^{1}$, hang $\mathrm{wu}^{1}$, and gaowu yan ${ }^{1}$ \\ ${ }^{1}$ Suining Central Hospital
}

May 5, 2020

\begin{abstract}
Eosinophilic gastroenteritis (EG) can easily be misdiagnosed. We present the case of a man with abdominal pain who was EG but misdiagnosed with cholelithiasis. This case highlights the need to consider EG in the differential diagnosis of abdominal pain since a favorable result can be achieved with timely treatment.
\end{abstract}

\section{Key Clinical Message}

Eosinophilic gastroenteritis is not only easy to ignore in clinical practice, but also easy to miss in the process of pathological diagnosis. There is a need to consider it in the differential diagnosis of alimentary disease.

Key words: Eosinophilic gastroenteritis; eosinophilic infiltration; cholelithiasis; abdominal pain.

\section{Introduction}

Eosinophilic gastroenteritis (EG) is a rare chronic inflammatory disease characterized by eosinophilic infiltration that may involve any part of the gastrointestinal (GI) tract wall with various manifestations [1,2]. Although no accurate epidemiologic data are available, the incidence of EG is estimated to be approximately $1-30 / 100,000[3,4]$. The stomach and small intestine are the most commonly affected areas. Based on the histopathological findings of eosinophilic infiltration, it can be divided into three types: mucosal, muscularis and subserosal [5]. Clinical manifestations vary greatly, depending on which layer and part of the GI tract are predominantly affected. Patients with the mucosal form mostly present with abdominal pain, vomiting, diarrhea, anemia, and protein-losing enteropathy. Obstructive symptoms are typical symptoms of the muscularis form, while the symptoms of the serosal forms may include ascites, bloating, possible peritonitis, and a high peripheral eosinophil count [6]. Due to its rarity and unspecific presentations, EG is not only easy to ignore in clinical practice, but also easy to miss in the process of pathological diagnosis [7], leading to misdiagnosis. Here, we present an EG case misdiagnosed as cholelithiasis and discuss its clinical characteristics. This can illustrate the importance of including EG in the differential diagnosis of abdominal pain even when there are no obvious allergens that suggest it as a possible reason.

\section{Case Report}

A 66-year-old man presented with a 15-day history of persistent upper abdominal pain and distension. The pain located in the middle and right upper abdomen. It was aggravated by drinking or eating. He had normal flatus and defection. No fever, vomiting, or hematemesis was reported. An unintentional weight loss of $2 \mathrm{~kg}$ in the last 15 days was reported. He reduced his food intake and took pantoprazole $40 \mathrm{mg}$ per day for three days. However, his condition did not improve. Therefore, he presented to the physicians of the local primary hospital because of unremitting distended pain. He was a farmer without a significant past 
medical history or a family history of malignancy. Physical examination was significant for slight distension in the upper middle and right abdomen, with normal bowel sounds and slight tenderness in the upper abdomen, especially in the right quadrant. No rebound was found. Laboratory studies showed a white blood cell (WBC) count of $14.5 \times 10^{9} / \mathrm{L}$, with $78.5 \%$ neutrophils, $7.5 \%$ eosinophils, a hemoglobin level of $13.4 \mathrm{~g} / \mathrm{dL}$, and a C-reactive protein (CRP) concentration of $48.3 \mathrm{mg} / \mathrm{L}$. Serum biochemical analyses, including glucose level, liver function, renal function, amylase, lipase, cardiac markers, and tumor markers, showed no abnormalities. Esophagogastroduodenoscopy (EGD) demonstrated diffuse thickening, focal mucosa erosions, hyperemia, and swelling of the antrum (Figure 1). Biopsies from the antrum showed mild chronic inflammation with some lymphocytes. Abdominal ultrasonography showed cholecystolithiasis (full gallstone) (Figure 4). Subsequently, cholecystectomy was performed successfully with the strong recommendation and insistence from his daughter who is a surgeon in the general department of the local hospital. The pathology showed chronic cholecystitis with some lymphocytes (Figure 5). The patient was observed overnight in the local hospital and was sent home the next morning.

However, the patient complained of more severe pain and distension after the surgery. The pain was gradually correlated with slight diarrhea with mucus and blood and tenesmus. Ten days after the surgery, the patient was seen in the emergency department of our hospital for progressive unremitting pain and distension. On physical examination, the abdomen was moderately distended, with active bowel sounds approximately 8-9 times per minute, diffuse tenderness without rebound and moderate ascites. Laboratory studies showed the following: hemoglobin level of $13 \mathrm{~g} / \mathrm{dL}$; PLT of $163 \times 10^{9} / \mathrm{L}$; WBC of $12.3 \times 10^{9} / \mathrm{L} ; 45.5 \%$ eosinophils; and albumin (ALB) $3.2 \mathrm{~g} / \mathrm{dL}$. Feces were positive for occult blood and negative for parasite ova.

Computer tomography (CT) of the abdomen showed thickening of the gastric antrum and sigmoid wall and a small amount of ascites in the pelvis (Figure 6). Colonoscopy showed diffuse thickening of mucosa, focal erythema and erosion (Figure 1), and biopsies showed eosinophilic infiltration of mucosa with more than 40 cells/HPF (Figure 3). Since obvious eosinophilic infiltration was found from the colonic mucosa, we doubted about the previous pathologic result of the EGD. So we rechecked the antrum biopsies which showed heavy infiltration of eosinophils: $>60$ cells/HPF (Figure 2). Ultrasound-guided diagnostic paracentesis showed a large number of eosinophilic granulocytes without malignant cells seen in punctate. For the evaluation of malignancy, the patient also underwent bone marrow aspiration and biopsy, the results of which were negative. Thus, the constellation of clinical presentation and histopathological findings were suggestive of eosinophilic gastroenteritis.

Subsequently, the patient received oral prednisone treatment at an initial dose of $40 \mathrm{mg} /$ day, combined with dietary restrictions, proton-pump inhibitors and mucosal protective agents. One week later, the patient noticed a marked improvement in his symptoms. The dosage of prednisone was gradually tapered off over an 8-week period. After the completion of the steroids, the patient's abdominal pain was completely relieved, and a peripheral blood count revealed an absolute normal eosinophil count level. Furthermore, CT imaging of the abdomen and pelvis showed a complete resolution of the gastric antrum and sigmoid thickening as well as ascites. Six months have elapsed since treatment, and the patient remains asymptomatic on no medications.

\section{Discussion}

Eosinophilic gastroenteritis (EG) is a chronic inflammatory disorder that is characterized by variable degrees of infiltration of eosinophils within the gastrointestinal tract. EG can occur at any age, and was first described by Kaijser in 1937 [8]. The true incidence of EG remains unclear due to its rare nature, and most of the literature is mainly case reports. Although no accurate epidemiologic data are available, the incidence of EG is estimated to be approximately 1-30/100,000 [3,4], and the prevalence seems to have been increasing in the past two decades [9]. Eosinophils are normally present in the lamina propria, except the esophagus, and the number of eosinophils along the GI tract varies, with the highest concentrations found in the cecum and appendix [10]. Eosinophils are involved in the mucosal immune system of the GI tract and play a role in host defense in healthy individuals [11]. The number of eosinophils increases during the pathogenesis of numerous inflammatory processes, including parasitic infections and allergic diseases. Then, the activated eosinophils 
produce and release highly bioactive inflammatory mediators, which may lead to a series of problems in the GI tract $[12,13]$. However, the exact mechanism of the pathogenesis of EG is still unclear.

Although the etiology and pathogenesis of EG remain unclear, there are studies suggesting that approximately 45-63\% of patients diagnosed with EG had a history of allergies, such as asthma, rhinitis, drug or food allergies; eczema; and parasitic infections [14,15], while others have found an association with other autoimmune conditions, such as HIV infection [16], influenza A virus infection [17], ulcerative colitis, and systemic lupus erythematosus [14]. The clinical manifestations of EG are not specific. The manifestations depend on the extent and depth of eosinophilic infiltration into the gastrointestinal tract wall. Three clinical forms are classified, mucosa, muscularis, and serosal, which are based on the Klein classification from 1970 [5]. The form involving the mucosal layer is the most common type, which can manifest as abdominal pain, hematochezia, diarrhea, hypoproteinemia and weight loss. Patients with EG that involves the muscularis can present with intestinal obstruction and even perforation. When the serosal layer is affected, ascites are the main manifestation. Full layer involvement is rare. Endoscopic findings may vary from nonspecific to mucosal erythema and ulceration [18]. Therefore, EG is easily misdiagnosed due to its various clinical and endoscopic manifestations.

In our case, we diagnosed the patient with eosinophilic gastroenteritis, and the full layer of the gastrointestinal wall was involved, mainly based on the following clinical evidence: First, signs of gastrointestinal whole wall involvement, such as diarrhea with blood and mucus, hypoproteinemia, abdominal pain, and abdominal distention, were observed. Second, endoscopic tissue biopsy revealed marked eosinophilic infiltration in the gastric antrum and sigmoid colon mucosa layer; eosinophils in peripheral blood and ascites increased significantly. Abdominal CT showed signs of thickened intestinal wall and ascites. Finally, the corresponding examination was improved to exclude tuberculosis peritonitis, IBD, parasitic infection, malignant tumor, intestinal Behcet's disease, lymphoma, and eosinophilia. Unfortunately, deep biopsies of the gastrointestinal tract were not available for additional evidence.

Corticosteroid therapy is the mainstay of EG treatment in both adults and children. Other therapies include dietary therapy, leukotriene inhibitors, mast cell stabilizers, interleukin-5 inhibitors, ketotifen, and biological agents $[13,19]$. We recommended methylprednisolone therapy to the patient, and the above symptoms were significantly relieved and eosinophils gradually decreased to normal. There was no recurrence after 6 months of follow-up.

Due to the lack of clinical experience, and little attention has been paid to the eosinophilic infiltration in the process of pathological diagnosis, doctors in primary hospitals misdiagnosed as cholelithiasis, and even cholecystectomy has been done. In conclusion, EG is a rare gastrointestinal disease with atypical clinical manifestations that are easily misdiagnosed. Timely and correct diagnosis depends on the clinician's understanding of the disease. Besides, more attention from the pathologists should be paid to the eosinophilic infiltration in the process of pathologic diagnosis. For patients with increased eosinophils in peripheral blood accompanied by gastrointestinal symptoms, EG should be considered. However, eosinophils in peripheral blood may not increase in some patients, which means further endoscopic multisite biopsy to determine whether eosinophils are infiltrating is the key to diagnosis.

\section{Conflict of interests}

The authors declare that they have no conflicts of interest.

\section{Author contributions}

LQ: served as the primary author and is responsible for this literature review and construction of the manuscript. HS: served as the final diagnosis as well as senior author managing the construction and edits of the manuscript and guiding the primary author through the submission process. Other authors have contributed in collecting the data for this manuscript.

\section{Acknowledgements}


The authors thank the patient involved for permitting the publication of the data regarding his case.

\section{References}

1. Abassa KK, Lin XY, Xuan JY, Zhou HX, Guo YW. Diagnosis of eosinophilic gastroenteritis is easily missed. World J Gastroenterol 23:3556-3564, 2017.

2. Liacouras CA, Furuta GT, Hirano I, Atkins D, Attwood SE, Bonis PA, Burks AW, Chehade M, Collins MH, Dellon ES. Eosinophilic esophagitis: updated consensus recommendations for children and adults. J Allergy Clin Immunol 128:3-20.e6; quiz 21-22, 2011.

3. Cianferoni A, Spergel JM. Eosinophilic Esophagitis and Gastroenteritis. Curr Allergy Asthma Rep 15:58, 2015.

4. Jensen ET, Martin CF, Kappelman MD, Dellon ES. Prevalence of Eosinophilic Gastritis, Gastroenteritis, and Colitis: Estimates From a National Administrative Database. J Pediatr Gastroenterol Nutr 62:36-42, 2016.

5. Klein NC, Hargrove RL, Sleisenger MH, Jef-fries GH: Eosinophilic gastroenteritis. Medicine (Baltimore) 49:299-319, 1970.

6. Zhang M, Li Y.Eosinophilic gastroenteritis: A state-of-the-art review. J Gastroenterol Hepatol 32:64$72,2017$.

7. Abassa KK, Lin XY, Xuan JY, Zhou HX, Guo YW. Diagnosis of eosinophilic gastroenteritis is easily missed. World J Gastroenterol 23:3556-3564, 2017.

8. Kaijser R. Zur Kenntnis der allergischen Affektionen des Verdauugskanals vom Standpunkt des Chirurgen aus. Arch Klin Chir 188:36-64, 1937.

9. Jensen ET, Martin CF, Kappelman MD, Dellon ES. Prevalence of eosinophilic gastritis, gastroenteritis, and colitis: estimates from a national administrative database. J Pediatr Gastroenterol Nutr 62:36-42, 2016.

10. Oh HE, Chetty R. Eosinophilic gastroenteritis: a review. J. Gastroenterol 43:741-750, 2008.

11. Khan S. Eosinophilic gastroenteritis. Best. Pract. Res. Clin. Gastroenterol 19: 177-198, 2005.

12. Hogan SP, Rothenberg ME, Forbes E, Smart VE, Matthaei KI, Foster PS. Chemokines in eosinophilassociated gastrointestin aldisorders. Curr. Allergy. Asthma. Rep 4:74-82,2004.

13. Zhang M, Li Y. Eosinophilic gastroenteritis: A state-of-the-art review. J Gastroenterol Hepatol.32:64$72,2017$.

14. Sunkara T, Rawla P, Yarlagadda KS, Gaduputi V.Eosinophilic gastroenteritis: diagnosis and clinical perspectives.Clin Exp Gastroenterol 5:239-253, 2019.

15. Wilechansky RM, Spring M, Huang Q, Zullow S.Eosinophilic esophagitis, gastroenteritis, and colitis in a patient with prior parasite exposure.Clin J Gastroenterol 12,530-533,2019.

16. Shrivastav O, Parikh NS, Jhaveri AB, Ahlawat R.Eosinophilic gastroenteritis: Atypical cause for chronic diarrhea in human immunodeficiency virus-associated immunosuppression. Indian J Sex Transm Dis AIDS 40:67-69, 2019.

17. Kim MJ, Oh MJ.Eosinophilic Enteritis Presenting as Massive Ascites after Influenza A Virus Infection in a Young Female. Korean J Gastroenterol 74:163-167, 2019.

18. Ashitani K, Tsuzuki Y, Yamaoka M, Ohgo H, Ichimura T, Kusano T, Nakayama T, Nakamoto H, Imaeda H. Endoscopic Features and Diagnostic Procedures of Eosinophilic Gastroenteritis. Internal Medicine 58:2167-2171, 2019.

19. Sasaki A, Sugimoto M, Tokaji N, Irahara M, Okamoto K, Uehara H, Kagami S. Efficacy of an elimination diet in a patient with eosinophilic gastroenteritis : a pediatric case with multiple food allergies. J Med Invest 66:201-204, 2019. 

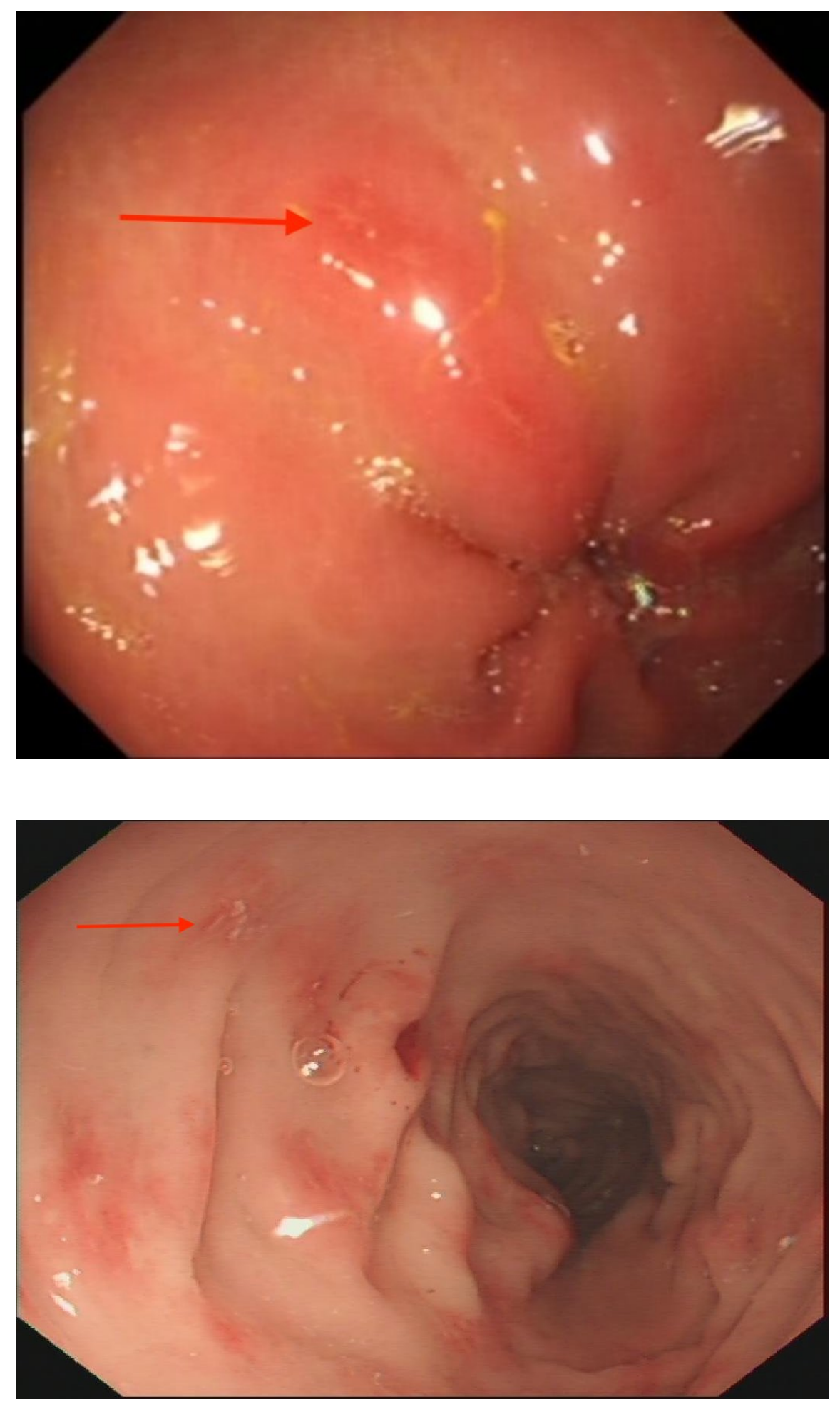

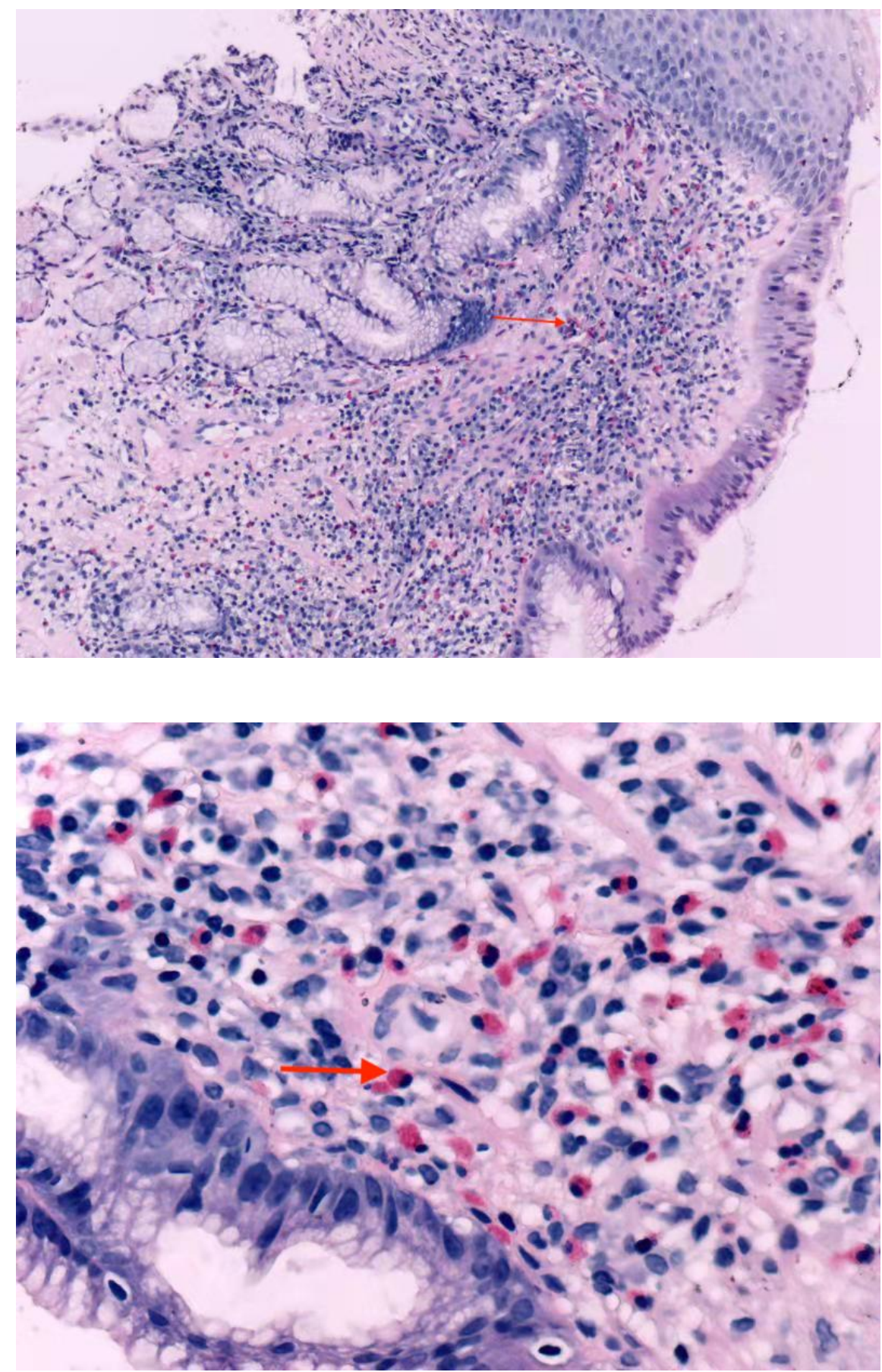

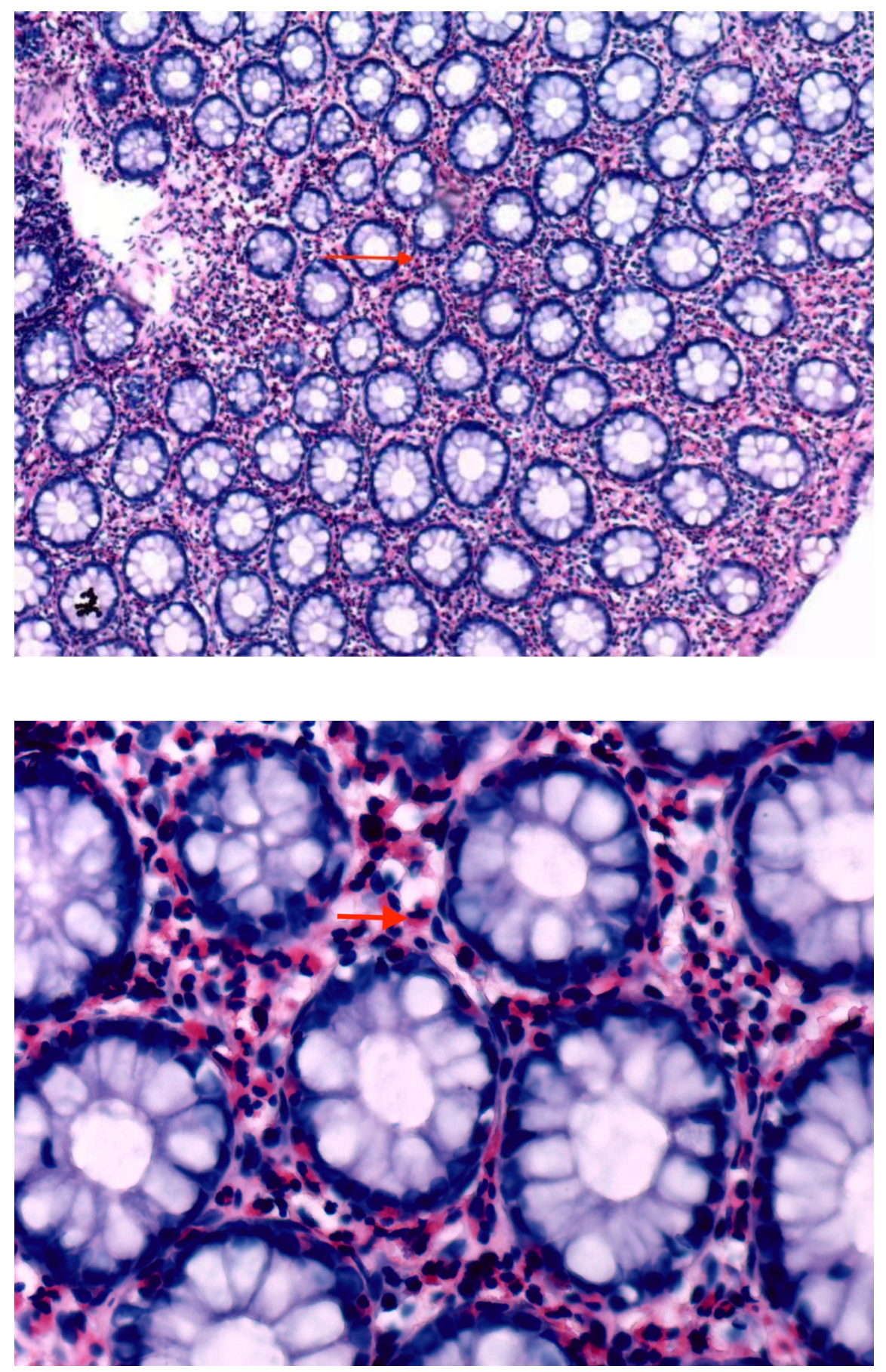

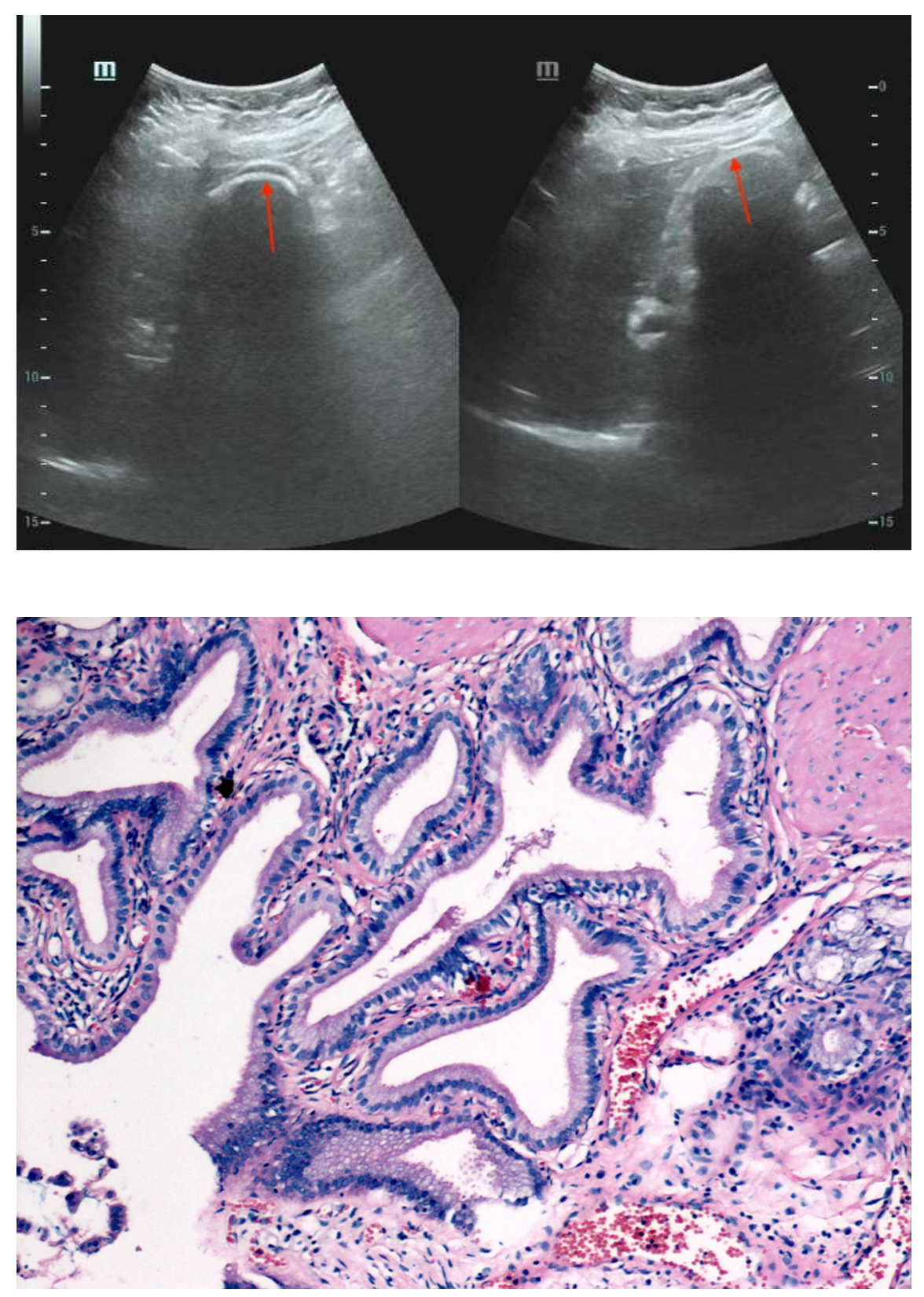

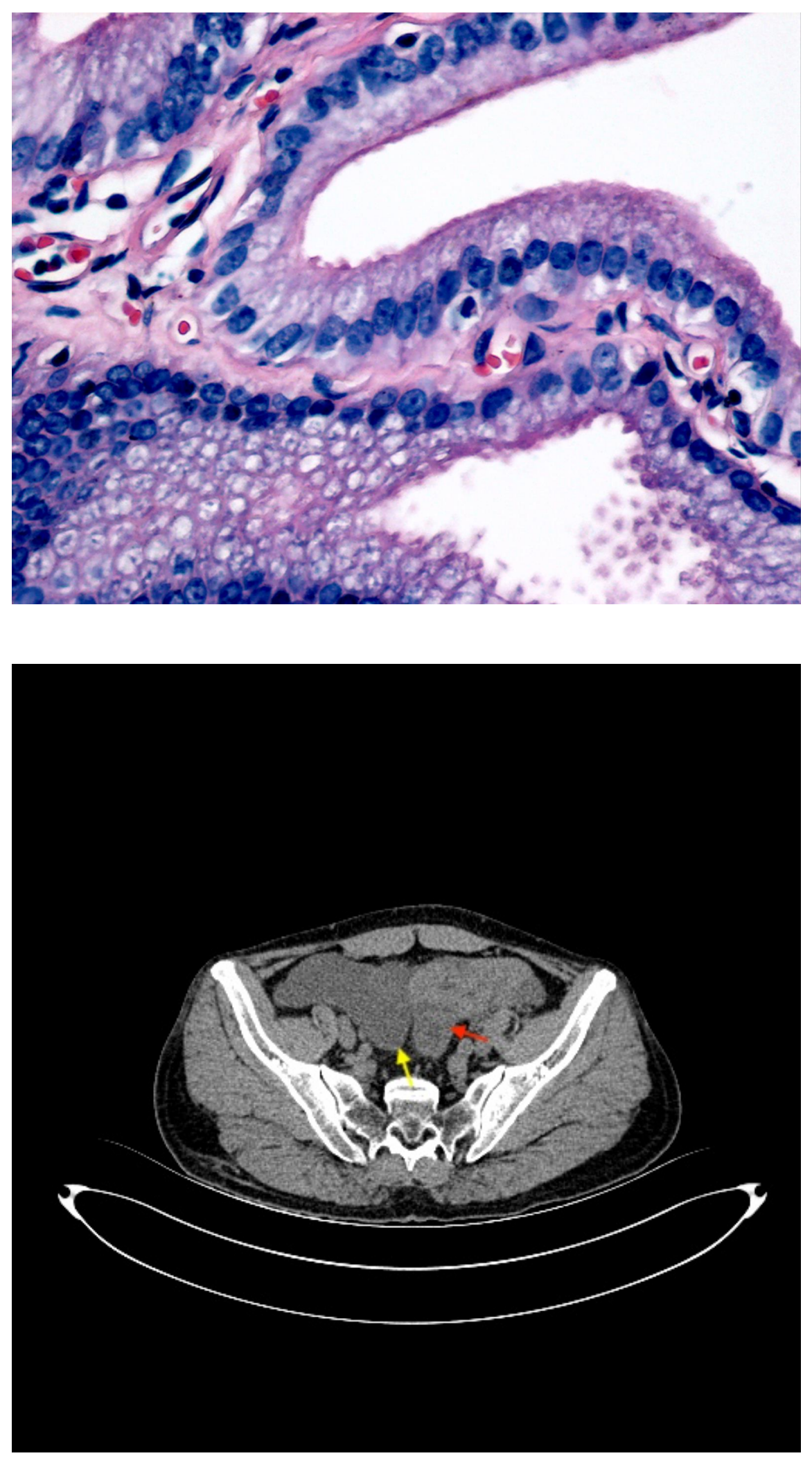